\title{
Enhanced ROI (Region of Interest Algorithms) for Medical Image Compression
}

\author{
Janaki. $R$ \\ Assistant Professor \& Head, Department of \\ Computer Science, N.K.R. Govt. Arts College for \\ Women, Namakkal - 637001
}

\author{
Dr. Tamilarasi. A \\ Professor \& Head, Department of Computer \\ Science, Kongu Engineering College, Perundurai
}

\begin{abstract}
Medical image compression applications are quality-driven applications which demand high quality for certain regions that have diagnostic importance in an image, where even small quality reduction introduced by lossy coding might alter subsequent diagnosis, which might cause severe legal consequences. Due to this, lossless techniques have been extensively used. As an alternative, owing to the observation that only some part of the image actually is of interest to the practitioners, ROI-based techniques are becoming popular. This paper proposes four techniques for this purpose. The four techniques are based on Mixed Raster Content layering, block-based thresholding, region growing and active contour algorithms. All the four techniques are enhanced and have the common objective of determining a ROI that can improve the compression process. Experiments results prove that all the four algorithms are efficient in determining the ROI and are efficient in terms of segmentation, compression and speed.
\end{abstract}

Keywords : ROI, MRC Layering, Block-based Segmentation, Region Growing, Active Contour.

\section{INTRODUCTION}

Medical imaging is an evolving and growing area of research and development both in academia as well as in industry. It involves interdisciplinary research and development encompassing diverse domains. New techniques and directions are being proposed in the literature every day. The medical equipments of today's modern era are creating huge number of high resolution images that are used by medical practitioners during analysis and diagnosis. These images while are revolutionizing the healthcare industry creates the problem of storage and transmission. For example, an image of size 512 x 512 pixels created by CT (Computed Tomography) requires about $1 / 4 \mathrm{MB}$ of storage space, thus stressing the need for image compression algorithms.

Image compression is the process of eliminating redundant data in an image in a fashion that minimizes the storage space requirement while maintaining the quality of the image. The algorithms used for this purpose are categorized as lossy and lossless, out of which lossless techniques are more popular in the medical domain. The reason behind this popularity is the need for recovering the decompressed image which is exactly the same as the original image. As healthcare professionals require accurate and clear picture, lossless techniques are not frequently used. Owing to the great demand for high compression ratio while maintaining high image quality, recently, Region of Interest (ROI) techniques have become acknowledged in medical compression [7]. The main advantage of using ROI-based compression techniques is that it combines the usage of both lossy and lossless techniques to compress images. Here, an image is initially segmented into two regions, interested and not-interested regions. It is assumed that the Interested Region (IR) consist of the most important part that has diagnostic/medicinal important, while the Not-Interested Region (NIR) has data that are not considered vital for diagnosis purposes. During compression, a lossless technique is used for IR while a lossy technique is used on NIR.

The method used for determining the ROI in medical images is still an active research area. The method used can be either manual or automatic, both with the same aim of achieving optimal compression balance between lossy and lossless regions. In this paper, different techniques are used to separate the IR and NIR regions of the medical images. All the techniques have the common aim of determining a ROI that can improve the compression process. Four techniques are proposed for IR and NIR segmentation. Lossy JPEG algorithm [15] and lossless JPEG algorithm [8] are used to compression the IR and NIR regions respectively. In this paper, the IR region represents the human organ and the NIR region represents the background of the acquired image. The rest of the paper is organized as follows. Section 2 presents a brief discussion on the existing medical image compression techniques. Section 3 presents the four enhanced ROI algorithms along with the compression model. The experimental results are discussed in Section 4 and the paper is concluded with future research directions in Section 5.

\section{LITERATURE STUDY}

There have been numerous compression research studies examining the use of compression to medical images. Most have focused on lossless algorithms since the medical community has been reluctant to adopt lossy techniques owing to the legal and regulatory issues that are raised, but this situation may start to change as more lossy research is performed. Popular traditional approaches used for encoding are Huffman encoding, Lempel-Ziv encoding, arithmetic encoding and run-length encoding [14]. Compression of medical images initially started with image preservation techniques like Scan pixel difference [13], followed by intra and inter-frame redundancy reduction [2]. $\mathrm{Hu}$ et al. [5] investigated linear predictive coding schemes. Several lossless compression techniques like Huffman coding, Lempel-Ziv coding, arithmetic coding have been proposed along with more recent coders like HINT (Hierarchical INTerpolation), DP (Difference Pyramid), Bit-Plane encoding and block truncation coding. All these techniques have concentrated on producing low compress rates. 
Transformation based coding schemes like Principal Component Analysis, Discrete Cosine Transform, Discrete Wavelet Transform have also been proposed to get better compression rates [16]. Till 2000, JPEG and Wavelet were most popular among medical community. These two compression methods actually gained widespread acceptance as lossy methods. However, each can also be made lossless which is the preferred style in medical imaging. Several studies have been proposed to compress medical images using wavelets and comparison of these techniques has also been studied.

Most of the studies have discussed the advantage of using wavelets for image compression. Most of the comparison studies have compared the performance of wavelets with JPEG coder [11]. Rahul et al. [9] compared wavelet coder with neural network based compression coder. Comparison between fractal based image compression and wavelets have also been analyzed ([3], [1]). Riazifar and Yazdi [10] analyzed the effectiveness of Contourlet and Wavelet Transform on Medical Image Compression.

\section{PROPOSED ROI TECHNIQUES AND COMPRESSION MODEL}

This section presents the four techniques that are used during the initial stage of ROI-based medical image compression. The four techniques are
(i) Layer-based segmentation
(ii) Block-based segmentation
(iii) Enhanced Region Growing Segmentation
(iv) Enhanced Active Contour-based Segmentation

\subsection{Layer-based Segmentation}

In layer-based approach, the original image is divided into rectangular and mask layers (planes). The rectangular layers represent the IR and NIR and the mask layer is used to specify which pixels of a particular layer should be included in the final composite image. After successful division, each layer is compressed with a specific compression method. As the compression model proposed needs to separate the medical image into two regions, a two layer-based approach is used in this paper. Most layered coding algorithms use the standard three layers Mixed Raster Content (MRC) representation ([6], [4]) and has wide usage in compound image compression domain. The main appeal of this approach is that it is the shortest path to supporting multiple region compression with existing standards. One main drawback of the traditional algorithm is the creation of halo effect on decompressed image, which affect the image quality. In this paper, this problem is solved using an average data filling method. The 3layer MRC model represents a color image as two multi-level layers (Foreground or IR, and Background or NIR) and one binary layer image (Mask or M). The mask layer describes how to reconstruct the final image from the IR/NIR layers (Equation 1)

$$
\operatorname{IMAGE}=(1-\mathrm{M}) * \mathrm{IR}+\mathrm{M} * \mathrm{NIR} \quad \mathrm{M} \in\{0,1\}
$$

Depending on the mask value on a certain position, a pixel from the IR or NIR on the corresponding position is selected (e.g. 0 for IR selection and 1 for NIR). Thus, the IR layer is poured through the mask onto the NIR layer. The imaging model, however, is composed of basic elementary plane (layer) pairs, IR and mask. Given a NIR, a IR plane is imaged onto it through the mask plane composing a new NIR image. The compressed layers are packaged in a format like TIFF-FX (file format for Internet Fax).
The MRC approach has two processes. The first process is plane decomposition and the second stage is the plane composition process. The plane decomposition is used to segment the image and the plan composition is used during decompression. The steps involved during decomposition and composition is shown in Figure 1. One consequence of MRC segmentation is that the layers produced after composition are actually sparse matrices and hence have missing parts which create a 'halo' effect. These are often termed as "don't care" regions. Each layer (IR or NIR) may contain unused pixels, since final pixels in some positions will be selected from the other layer. Thus, these pixels can be replaced by any color in order to enhance compression [17]. Redundant regions are marked in each IR/NIR layer and the main goal here is to replace the redundant data with other data that will enhance compression.

The technique discussed for this purpose can be used to process both IR or NIR layers and the steps are given below.

Step 1: Divide the image into blocks of size $8 \times 8$.

Step 2: Blocks lying entirely in the NIR are left intact.

Step 3: Blocks lying entirely in the IR are filled with the overall image mean.

Step 4: Partially empty blocks are then filled by using an iterative approach that propagates values from the existing pixels, using overall image mean value.

The proposed compression model that uses the enhanced MRC-based ROI segmentation has the following steps.

Step 1: Segment the image into IR and NIR layers

Step 2: Create the binary mask layer

Step 3: Apply data filling to remove the halo effect

Step 4 : Compress IR using lossless JPEG, NIR using lossy JPEG and mask layer using JBIG compressors.

Step 5: Combine results into one meaningful stream for transmission or storage.

After identifying the IR and NIR regions along with the binary mask layer, the IR and NIR regions are compressed using lossless and lossy JPEG coders respectively. The JBIG (Joint Bi-level Image Experts Group) algorithm is used to losslessly compress the mask layer. The mask layer contains only two values ' 0 ' or ' 1 ' to indicate whether a pixel is taken from IR block or background block in MRC layering.

As JBIG is a method for compression bi-level image data, it is considered as the right candidate to compress 


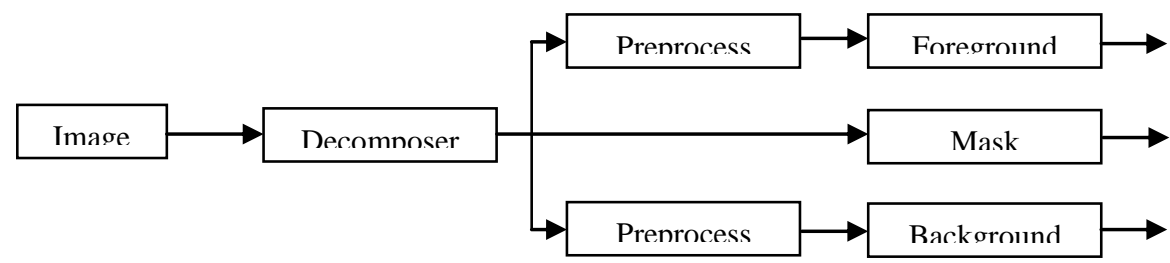

(a) Plane Decomposition Process

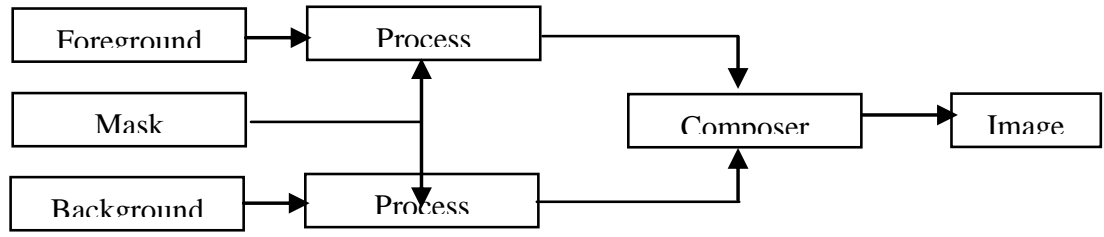

(b) Plane Composition Process

Figure 1 : MRC Process

the mask layer. JBIG encodes redundant image data by comparing a pixel in a scan line with a set of pixels already scanned by the encoder.

These additional pixels are called a template and they form a simple map of the pattern of pixels that surround the pixel that is being encoded. The values of these pixels are used to identify redundant patterns in the image data. These patterns are then compressed using an adaptive arithmetic compression coder. JBIG is capable of compressing color or grayscale images up to 255 bits per pixel.

\subsection{Block-based Segmentation}

The proposed block-based segmentation technique uses a block classification algorithm to segment the input image into IR and NIR. Initially, the block-based segmentation technique divides the image into $16 \times 16$ sized blocks. The classification algorithm is based on two features, namely, histogram and gradient of the block. The pixels of each block are grouped into three classes, namely, low-gradient pixels, mid-gradient pixels and high-gradient pixels, according to pixel's gradient value. Then the histogram distribution for each pixel group is computed. This method is based on the fact that the gradienthistogram distribution of IR and NIR is different.

$$
\begin{aligned}
& \text { For } \mathrm{B}=1 \text { to } \mathrm{N} \\
& \text { Classify pixels as low, mid and high gradient pixels } \\
& \text { Compute histogram distribution for each pixel in each } \\
& \text { group } \\
& \text { If High gradient pixels + low gradient pixels < } \\
& \text { B is IR block } \\
& \text { Else if (High gradient pixels }<\mathrm{T} 2 \text { \& \& low } \\
& \text { gradient pixels }>\text { T3) then } \\
& \text { If Number of colour level }<\mathrm{T} 4 \text { then } \\
& \text { Else } \quad \text { B is NIR block } \\
& \text { End } \quad \text { B is IR block }
\end{aligned}
$$

Figure 2 : Block Based Segmentation
The NIR blocks typically contain only low gradient pixels and show one peak at the low-gradient histogram. On the other hand the IR region shows several peaks at the mid-gradient and high-gradient histograms. Each block is identified using a block map which is compressed using an Arithmetic coder. The block-based segmentation is given in Figure 2, where B denotes a block and $\mathrm{N}$ denotes the number of $16 \times 16$ blocks in an image and after many tests, the thresholds $T_{1}, T_{2}, T_{3}$ and $\mathrm{T}_{4}$ were set as 50, 45, 10 and 2 respectively.

After segmentation, the IR region is compressed using lossless JPEG and NIR region is compressed using lossy JPEG coder. The block Map is compressed using an Arithmetic coder.

\subsection{Enhanced Region-Growing Segmentation}

The goal of region growing segmentation algorithm is to group regions having common properties between a pixel and its neighbour. The properties can be intensity values of the original image or unique texture patterns of each region or spectral profiles that provide multidimensional image data. The algorithm provides multiple merits during segmentation. The borders of regions found by region growing are perfectly thin and well connected.

The algorithm is very stable with respect to noise. Most importantly, membership in a region can be based on multiple criteria. It is possible to take advantage of several image properties, such as low gradient or gray level intensity value, at once, while using region growing algorithm. The traditional region growing algorithm has two major issues. The first is the selection of initial seed points. Incorrect selection leads to inaccurate segmentation and therefore an automatic process is always preferred. The second is that even with automated process, the selected seed point may lie on an edge. In this paper, the steps in Figure 3 is used to solve both the issues. The last step in the algorithm consist of two conditions that examine the candidate pixels and makes sure that the selected seed point is highly similar to its neighbour and is not a boundary region. For this purpose, the relative Euclidean distance [12] between the seed point and its neighbours is calculated using Equation 2.

$$
\mathrm{d}_{\mathrm{i}}=\frac{\sqrt{\mathrm{P}-\mathrm{NP}_{\mathrm{i}}}}{\sqrt{\mathrm{P}^{2}}}
$$


where $\mathrm{P}$ denotes the seed point and $\mathrm{i}$ denotes its 8 neighbouring pixels. After integrating the automated process of initial seed selection, the enhanced region growing algorithm consists of the following steps.

- Divide the image into $16 \times 16$ blocks

- For each block, $\mathrm{i},(1 \geq \mathrm{i} \leq \mathrm{N})$

- Calculate threshold T

1. Initialize $\mathrm{T}$ as average of maximum and minimum intensity

2. Repeat Steps 3 to 5 till T converges

3. Group pixels into two groups, G1 and G2, where G1 has pixels whose intensity value is greater than T and G2 has pixels whose intensity value is less than $\mathrm{T}$

4. Calculate Mean ( $\mu 1$ and $\mu 2)$ and Standard Deviation $(\sigma 1$ and $\sigma 2)$ of G1 and $\mathrm{G} 2$ respectively

5. Re-estimate $\mathrm{T}, \mathrm{T}=0.5 *[(\mu 1$ and $\mu 2)+$ $(\sigma 1$ and $\sigma 2)]$ and goto step 3

- Calculate total variance (TV) and mean variance (MV)

- $\quad$ Calculate Seed Threshold, $\mathrm{T}_{\mathrm{S}}=\mathrm{TV}+\mathrm{MV}$

- Determine pixels with $T_{\mathrm{s}}<\mathrm{T}$ and select them as initial candidate seed points

- Selected seed point only if both the conditions listed below are satisfied

1. The candidate seed pixel point must have the similarity higher than $\mathrm{T}_{\mathrm{S}}$

2. The maximum relative Euclidean distance to the candidate seed's eight neighbours should be less than $\mathrm{T}_{\mathrm{S}}$

\section{Figure 3 : Automatic Selection of Initial Seeds}

1. Apply automatic seed selection algorithm to obtain initial seeds for region growing

2. Calculate distance between seed point and its neighbours

3. Check the neighboring pixels and add them to the region if they are similar to the seed

4. Repeat steps 2 and 3 until no more pixels can be added.

\subsection{Enhanced Active Contour based Segmentation}

In active contour based segmentation algorithm, the user specifies an initial guess for the contour, which is then moved by image driven forces to the boundaries of the desired objects. The idea behind active contours, or deformable models, for image segmentation is quite simple. Using the user specified initial guess, the contour is moved by image driven forces to the boundaries of the desired objects. In such models, two types of forces are considered - the internal forces, defined within the curve, are designed to keep the model smooth during the deformation process, while the external forces, which are computed from the underlying image data, are defined to move the model toward an object boundary or other desired features within the image.

The main challenge while using active contour models for both segmentation is the initial seed selection. Different initial seed values leads to different segmentation result and often incorrect selection produces inaccurate segmentation. To solve this problem, this paper proposes the use of region growing algorithm first to estimate the initial seeds which are then used by the active contour model.

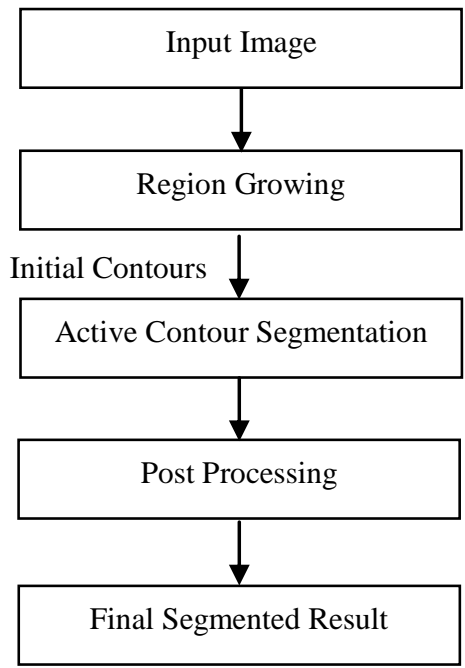

Figure 4 : Enhanced Active Contour

The growing parameter adopted is the average between the maximal and minimal intensities of the input image. A post processing step that performs region merging to merge small regions is included to improve the segmentation result.

This method merges several small segments and isolates image background by considering the distance between region's intensity. All groups with similar intensities are grouped together. The algorithm is illustrated in Figure 4.

\section{EXPERIMENTAL RESULTS}

Experimental evaluation was performed using a series of benchmark images (Figure 5). The ROI segmentation was evaluated in two stages. The first stage evaluated the performance of the proposed ROI algorithms and the second stage analyzed the effect of ROI algorithms on compression. The quality metrics used during performance evaluation are compression efficiency in terms of bits per pixel, Peak Signal to Noise Ratio (PSNR), time complexity of both ROI and compression process and visual comparison of the results. All the experiments were conducted using a Pentium IV machine with $512 \mathrm{MB}$ RAM and the implementation was done in MATLAB 2009a. The compression results are compared with the traditional JPEG lossless algorithm.

In the projected results, MRC-T, BLK-T, REG-T and ACT-T represent the enhanced MRC-based algorithm, block-based thresholding algorithm, enhanced region growing algorithm and enhanced active contour based algorithm respectively.

\subsection{ROI-Segmentation Results}

The result after ROI separation is shown in Figure 5. Table 1 shows the time taken by each of the proposed algorithms to segment the IR from the original image. 
Table 1 : Time Efficiency of the ROI Algorithms

\begin{tabular}{|l|c|c|c|c|}
\hline \multicolumn{1}{|c|}{ Images } & MRC-T & BLK-T & REG-T & ACT-T \\
\hline CT.jpg & 0.38 & 0.34 & 0.36 & 0.32 \\
\hline MRI.jpg & 0.17 & 0.21 & 0.12 & 0.09 \\
\hline Radio.jpg & 0.12 & 0.15 & 0.09 & 0.08 \\
\hline
\end{tabular}

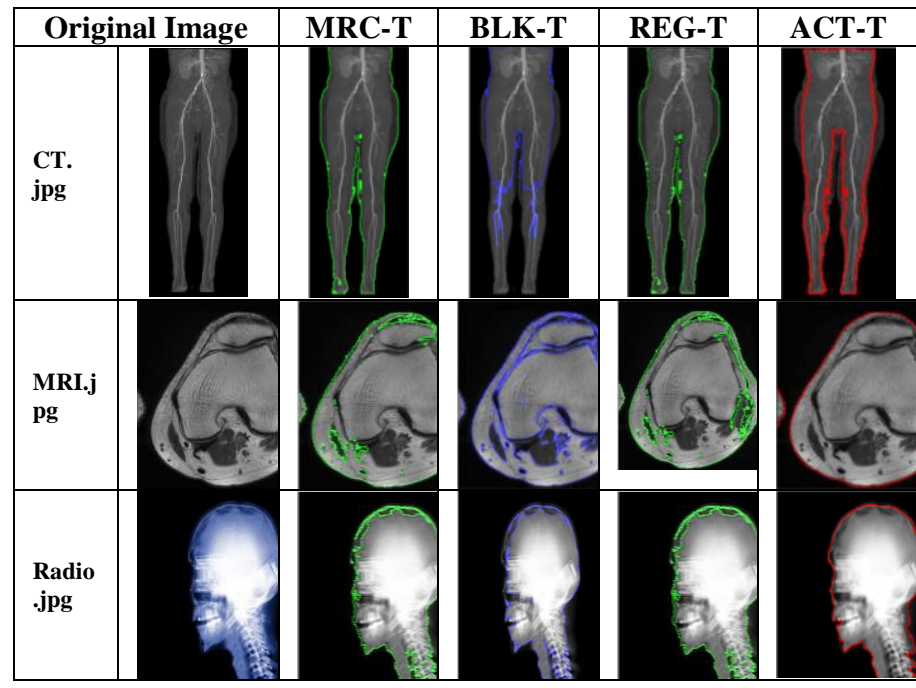

Figure 5 : Segmentation Results

From stage I experiments, it is evident that the enhanced active contour ROI algorithm is efficient in separating the IR and NIR regions of an image (Figure 5). This is followed by the enhanced region growing algorithm and MRC-layering based segmentation algorithm. Out of the four proposed technique, the block based algorithm showed significant decrease in performance, which might be due to its sensitivity to manual initialization of the threshold values. While considering the speed of the proposed ROI segmentation algorithms (Table 1), again the active contour-based algorithm enhanced with region growing initial seed estimation and post processing proved to be the fastest among the four proposed algorithm. Again the trend proved that the block-based segmentation technique is the slowest.

\subsection{Compression Results}

The four ROI segmentation algorithms were evaluated in terms of compression efficiency and is shown is Table 2 . The experiment used lossy JPEG and lossless JPEG to compress IR and NIR region. An JBIG algorithm was used to compress the mask layer of MRC segmentation and arithmetic coder was used to compress the block map of the block-based segmentaton algorithm.

Table 2 : Compression Efficiency (bpp)

\begin{tabular}{|l|c|c|c|c|c|}
\hline Image & $\begin{array}{c}\text { MRC- } \\
\text { T }\end{array}$ & $\begin{array}{c}\text { BLK- } \\
\text { T }\end{array}$ & $\begin{array}{c}\text { REG- } \\
\text { T }\end{array}$ & $\begin{array}{c}\text { ACT- } \\
\text { T }\end{array}$ & JPEG \\
\hline CT.jpg & 1.241 & 1.232 & 1.226 & 1.244 & 1.218 \\
\hline MRI.jpg & 8.501 & 8.309 & 8.384 & 8.532 & 8.278 \\
\hline Radio.jpg & 1.299 & 1.289 & 1.274 & 1.301 & 1.251 \\
\hline
\end{tabular}

From the table, it is clear that all the four algorithms show significant compression improvement when compared to JPEG. While comparing the proposed four algorithms, the active contour model ranks first, followed by MRC algorithm and block based segmentation algorithm. The region growing algorithm shows decreased compression efficiency in terms of compression gained in terms of bits per pixels.
Table 3 shows the Peak Signal to Noise Ratio obtained for the original and decompressed images.

Table 3 : PSNR (dB)

\begin{tabular}{|l|c|c|c|c|c|}
\hline Image & MRC- & $\begin{array}{c}\text { BLK- } \\
\text { T }\end{array}$ & $\begin{array}{c}\text { REG- } \\
\text { T }\end{array}$ & $\begin{array}{c}\text { ACT- } \\
\text { T }\end{array}$ & JPEG \\
\hline CT.jpg & 27.82 & 27.02 & 27.69 & 28.10 & 26.98 \\
\hline MRI.jpg & 37.16 & 34.59 & 35.91 & 37.82 & 33.75 \\
\hline Radio.jpg & 38.04 & 37.13 & 37.55 & 38.66 & 36.01 \\
\hline
\end{tabular}

The results in Table 2 again conform that the usage of ROI segmentation to compress medical images is positive as evident from the higher PSNR values obtained when compared to JPEG algorithm. The enhanced active contour model again outperforms the rest of the three algorithms.

Table 4 shows the time taken for compressing the images. The compression and decompression time is the execution time taken by the system to perform the compression and decompression processes. The total compression time is calculated as the sum of compression time and decompression time.

Table 4 : Compression Time

\begin{tabular}{|l|c|c|c|c|c|}
\hline Images & $\begin{array}{c}\text { MRC- } \\
\text { T }\end{array}$ & $\begin{array}{c}\text { BLK- } \\
\text { T }\end{array}$ & $\begin{array}{c}\text { REG- } \\
\text { T }\end{array}$ & $\begin{array}{c}\text { ACT- } \\
\text { T }\end{array}$ & JPEG \\
\hline CT.jpg & 0.598 & 0.661 & 0.504 & 0.499 & 0.420 \\
\hline MRI.jpg & 0.206 & 0.252 & 0.197 & 0.185 & 0.133 \\
\hline Radio.jpg & 0.150 & 0.182 & 0.139 & 0.128 & 0.112 \\
\hline
\end{tabular}

From the above data, it is clear that the trend of performance with respect to compression speed has changed and JPEG algorithm is considerably faster than the four proposed compression models. This result is obvious as the proposed algorithm includes a ROI segmentation process. However, the efficiency gain obtained with respect to compression ratio and visual quality encourages to the use of the proposed compression models. The visual comparison of the compression result is shown in Figure 6.

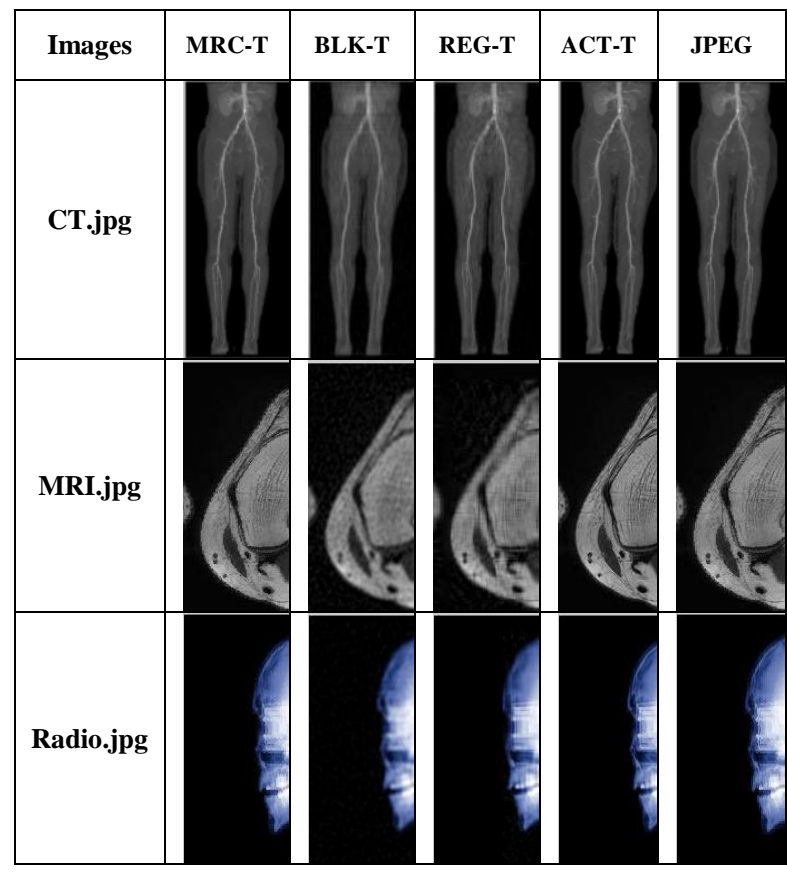

Figure 6 : Compression Results 
From the results obtained it is clear that the inclusion of ROI segmentation yields efficiency compression and produces good image quality after decompression and therefore can be considered suitable by many medical imaging systems.

\section{CONCLUSION}

Medical image compression applications are quality-driven applications which demand high quality for certain regions that have diagnostic importance in an image, where even small quality reduction introduced by lossy coding might alter subsequent diagnosis, which might cause severe legal consequences. Due to this, lossless techniques have been extensively used. As an alternative, owing to the observation that only some part of the image actually is of interest to the practitioners, ROI-based techniques are becoming popular. This paper proposes four techniques for this purpose. The first method uses the Master-Raster Content layering based segmentation approach enhanced to use an average block averaging algorithm to avoid the halo effect. The second method is an enhanced block-based thresholding algorithm, while the third technique is an enhanced version of the traditional region growing algorithm. The fourth method enhanced the active contour model to separate the input image into IR and NIR. Various experiments on the performance of segmentation and compression revealed that the enhanced active contour model followed by MRC based segmentation and region growing algorithm is efficient and can be considered as a promising candidate by medical imaging systems. In future, tailor-made methods for lossless and lossy compression of the IR and NIR images are to be designed and tested with the proposed ROI algorithms.

\section{REFERENCES}

[1] Aggarwal, P. and Rani, B. (2010) Performance Comparison of Image Compression Using Wavelets, International Journal of Computer Science and Communication, Vol. 1, No. 2, Pp. 97-100.

[2] Assche, S.V., Rycke, D.D., Philips, W. and Lemahieu, I. (2000) Exploiting interframe redundancies in the lossless compression of 3D medical images, Data Compression Conference, P. 575.

[3] Duraisamy, R., Valarmathi, L. and Ayyappan, J. (2008) Iteration Free Hybrid Fractal Wavelet Image Coder, International Journal of Computational Cognition, Vol. 6, No. 4, Pp. 34-40.

[4] Eri, H., YI, J. and Charles A,B. (2007) Segmentation for MRC compression, Proceedings of SPIE, The International Society for Optical Engineering, Color imaging. Conference, Vol.6493, San Jose, California, USA)

[5] Hu, J., Wang, Y. and Cahill, P.T. (1997) Multispectral code excited linear prediction coding and its application in magnetic resonance images, IEEE Transactions on Image Processing, Vol. 6, No. 11, Pp. 1555 -1566.

[6] ITU-T Recommendation T.44 (1998) Mixed Raster Content (MRC), Study Group-8 Contribution.
[7] Palanisamy, G. and Samukutti, A. (2008) Medical image compresssion using a novel embedded set partitioning significant and zero block coding, The International Arab Journal of Information Technology, Vol. 5, No. 2, Pp. 132-139.

[8] Pennebaker, W.B. and Mitchell, J.L. (1993) JPEG Still Image Data Compression Standard. New York: Van Nostrand Reinhold.

[9] Rahul, S., Vignesh, J., Santhosh Kumar, S., Bharadwaj, M. and Venkateswaran, N. (2007) Comparison of Pyramidal and Packet Wavelet Coder for Image Compression Using Cellular Neural Network (CNN) with Thresholding and Quantization, International Conference on Information Technology (ITNG'07), Pp.183-184.

[10] Riazifar, N. and Yazdi, M. (2009) Effectiveness of Contourlet vs Wavelet Transform on Medical Image Compression: a Comparative Study, World Academy of Science, Engineering and Technology, Vol. 49, Pp.837842.

[11] Saffor, A., Ng, K.H., Ramli, A.R. and Dowsett, D. (2002)A Comparison of JPEG and Wavelet Compression Applied to Computed Tomography Brain, Chest, and Abdomen Images, The Internet Journal of Medical Simulation and Technology, Vol. 1, No. 1.

[12] Shih, F.Y. and Cheng, S. (2005) Automatic seeded region growing for color image segmentation, Image and Vision Computing, Science Direct, Vol. 23, Pp. $877-$ 886.

[13] Takaya, K. and Tannous, C.G. (1995) Information preserved guided scan pixel difference coding for medical images, WESCANEX 95, Communications, Power, and Computing, IEEE Conference Proceedings., Vol. 1 , Pp. $238-243$.

[14] Tsung-Ching, L., Chien-Wen, C., Shi-Huang, C. and Trieu-Kien, T. (2008) Near lossless medical image compression using JPEG-LS and cubic spline interpolation, Optics and Photonics for Information Processing II. Edited by Awwal, Abdul Ahad Sami; Iftekharuddin, Khan M.; Javidi, Bahram. Proceedings of the SPIE, Volume 7072, pp. 70721G-70721G-7.

[15] Weinberger, M.J., Seroussi, G. and Sapiro, G (2000) The LOCO-I lossless image compression algorithm: principles and standardization into JPEG-LS, IEEE Transactions on Image Processing, Vol. 9, Issue 8, Pp.1309-1324.

[16] Yoshioka, M. and Omatu, S. (1996) Image Compression by nonlinear principal component analysis, IEEE Conference on Emerging Technologies and Factory Automation, EFTA '96,Vol. 2, Pp. 704 -706.

[17] Zaghetto, A. and de Queiroz, R.L. (2008) Iterative preand post-processing for MRC layers of scanned documents, 15th IEEE International Conference on Image processing, ICIP 2008, Pp. 1009-1012. 